\title{
Serum Mannose-Binding Lectin Levels Are Correlated with the Disease Activity of Antineutrophil Cytoplasmic Antibody- Associated Vasculitis: A Single-Center Study
}

\author{
Taejun Yoon, ${ }^{1, *}$ Sung Soo Ahn, ${ }^{2, *}$ Juyoung Yoo, ${ }^{2}$ Byung-Woo Yoo, ${ }^{2}$ \\ Hyeok Chan Kwon, ${ }^{2}$ Seung Min Jung, ${ }^{2}$ Jason Jungsik Song, ${ }^{2,3}$ Yong-Beom Park ${ }^{2,3}$ \\ and Sang-Won Lee Le, $^{2,}$ \\ ${ }^{1}$ Department of Medical Science, BK21 Plus Project, Yonsei University College of Medicine, Seoul, Republic of \\ Korea \\ ${ }^{2}$ Division of Rheumatology, Department of Internal Medicine, Yonsei University College of Medicine, Seoul, \\ Republic of Korea \\ ${ }^{3}$ Institute for Immunology and Immunological Diseases, Yonsei University College of Medicine, Seoul, Republic \\ of Korea
}

\begin{abstract}
Mannose-binding lectin (MBL) is a soluble pattern-recognition molecule, which plays a crucial role in the innate immune system and the activation of lectin complement pathway. Antineutrophil cytoplasmic antibody (ANCA)-associated vasculitis (AAV) is an autoimmune disease affecting the small vasculatures and is characterized by the alteration of innate and adaptive immunity and complement activation. In this study, we investigated whether serum MBL is associated with disease activity of AAV, which was measured by ELISA. Associations between serum MBL and AAV-specific indices, as well as clinical and laboratory data were assessed using Kendall's tau. Among the 80 patients, 42 (52.5\%), 21 (26.3), and $17(21.3 \%)$ patients were classified as microscopic polyangiitis (MPA), granulomatosis with polyangiitis (GPA), and eosinophilic granulomatosis with polyangiitis (EGPA), respectively. The median values of erythrocyte sedimentation rate, C-reactive protein, and serum MBL were 36.5 (normal range $<20$ ) $\mathrm{mm} / \mathrm{h}, 2.4$ (normal range < 8) $\mathrm{mg} / \mathrm{dL}$, and $8.6 \mathrm{ng} / \mathrm{mL}$, respectively. The median serum levels of MBL in MPA, GPA, and EGPA patients were $8.4,9.3$, and $8.2 \mathrm{ng} / \mathrm{mL}$. Correlation analysis showed that serum $\mathrm{MBL}$ was associated with Birmingham Vasculitis Activity Score (BVAS) $(R=0.169, p=0.027)$, but not with other AAV-specific indices and clinical and laboratory data. In addition, serum MBL was significantly associated with the pulmonary manifestation score based on BVAS $(R=0.247, p=0.001)$. In summary, among the AAV-specific indices and clinical and laboratory variables analyzed, serum MBL is correlated with BVAS and pulmonary manifestation score based on the BVAS.
\end{abstract}

Keywords: antineutrophil cytoplasmic antibody; Birmingham Vasculitis Activity Score; disease activity; mannosebinding lectin; vasculitis

Tohoku J. Exp. Med., 2020 June, 251 (2), 117-123.

\section{Introduction}

Antineutrophil cytoplasmic antibody (ANCA)associated vasculitis (AAV) is an autoimmune disease affecting the small vasculatures which is characterized by the alteration of innate and adaptive immunity and complement activation (Chen et al. 2017). According to the 2012
Chapel Hill Consensus Conferences Nomenclature of Vasculitis (the 2012 CHCC definitions), AAV is classified into three different disease subtypes: microscopic polyangiitis (MPA), granulomatosis with polyangiitis (GPA), and eosinophilic granulomatosis with polyangiitis (EGPA) based on the clinical and histologic findings (Jennette et al. 2013b). Generally, a different pattern of organ involvement

\footnotetext{
Received October 10, 2019; revised and accepted June 5, 2020. Published online June 23, 2020; doi: 10.1620/tjem.251.117.

*These two authors contributed equally to this work.

Correspondence: Sang-Won Lee, M.D., Ph.D., Division of Rheumatology, Department of Internal Medicine, Yonsei University College of Medicine, 50-1 Yonsei-ro, Seodaemun-gu, Seoul 03722, Republic of Korea.

e-mail: sangwonlee@yuhs.ac

(C)2020 Tohoku University Medical Press. This is an open-access article distributed under the terms of the Creative Commons Attribution-NonCommercial-NoDerivatives 4.0 International License (CC-BY-NC-ND 4.0). Anyone may download, reuse, copy, reprint, or distribute the article without modifications or adaptations for non-profit purposes if they cite the original authors and source properly.

https://creativecommons.org/licenses/by-nc-nd/4.0/
} 
is present according to the AAV subtypes. MPA mainly induces necrotizing and crescentic glomerulonephritis and pulmonary capillaritis, whereas GPA is usually characterized by granulomatosis in the upper and lower respiratory tracts and occasional glomerulonephritis. On the other hand, EGPA typically exhibits three allergic features, including asthma, peripheral eosinophilia, and eosinophilrich granuloma in the respiratory tracts (Jennette et al. 2013b; Kallenberg 2014).

The complement system plays a critical role in mediating the immune system and is activated via three pathways of the classical, alternative, and lectin pathway. Notably, mannose-binding lectin (MBL), which is produced by hepatocytes, is involved in the activation of the lectin complement pathway (Turner 2003). Briefly, multimers of MBL interacts with MBL-associated serine proteases (MASPs) (Roos et al. 2007), and the formation of MBL-MASP complex finally cleaves complement (C) 5 into C5a and C5b. Whereas $\mathrm{C} 5 \mathrm{~b}$ is known to form a membrane attack complex with $\mathrm{C} 6, \mathrm{C} 7, \mathrm{C} 8$, and $\mathrm{C} 9$ to induce cytolysis, C5a may exhibit anaphylactic activity and provoke systemic inflammation in various diseases (Pagowska-Klimek and Cedzynski 2014). In addition, MBL has been implicated in the innate immune system by directly acting as a soluble pattern-recognition molecule prior to the activation of adaptive immune response (Ezekowitz 2003; Takahashi and Ezekowitz 2005).

In the pathogenesis of $\mathrm{AAV}$, the production of $\mathrm{C} 5 \mathrm{a}$ as a result of complement pathway activation promotes neutrophil mediated vessel inflammation (Jennette et al. 2013a; Kallenberg et al. 2013). In line with this finding, a previous study by Yuan et al. (2012) has demonstrated that elevated plasma and urinary C5a levels indicate higher disease activity in AAV. Therefore, given that C5a presents a link between MBL-mediated immunological functions and AAV pathogenesis, it can be assumed that serum MBL might be associated with disease activity or organ injury in AAV. In particular, even though a previous study has demonstrated that MBL gene polymorphisms are not associated with AAV (Kamesh et al. 2007), the clinical significance of serum MBL in AAV has not been well described. Hence, the objective of this study was to investigate whether serum MBL is associated with disease activity or organ injury in patients with AAV.

\section{Methods}

\section{Patients}

This study included 80 patients with AAV from the Severance Hospital ANCA-associated VasculitidEs (SHAVE) cohort from the period of November 2016 to May 2019. The SHAVE cohort is a prospective cohort of AAV patients, which started in November 2016. All patients enrolled in the SHAVE cohort undergo laboratory and imaging tests on every visit, and the assessment of AAVspecific indices and blood sample collection was performed every three to six months. Sera were isolated from the col- lected blood samples and then stored at $-70^{\circ} \mathrm{C}$ until further use. All patients fulfilled the 1990 American College of Rheumatology classification criteria (the 1990 ACR criteria) (Leavitt et al. 1990; Masi et al. 1990), and was classified based on the 2007 European Medicines Agency algorithms (Watts et al. 2007) and the 2012 CHCC definitions (Jennette et al. 2013b). This study was approved by the Institutional Review Board of Severance Hospital (4-20160901) and written informed consents were obtained from all patients at the time of blood sampling.

\section{Definition of AAV-specific indices}

AAV-specific indices included Birmingham Vasculitis Activity Score (BVAS) (version 3), Five-Factor Score (FFS), Vasculitis Damage Index (VDI), and Short Form 36-Item Health Survey (SF-36) (Han et al. 2004; Mukhtyar et al. 2009; Guillevin et al. 2011; Bhamra and Luqmani 2012). BVAS was used as an index to assess the activity and severity of AAV, and FFS and VDI was adopted as a prognostic and damage index. The Korean version of the SF-36 was utilized as a functional index. In addition, because Birmingham Vasculitis Activity Score for Granulomatosis with Polyangiitis (BVAS/GPA) is used specifically in GPA and has a different weighting system compared to BVAS (Stone et al. 2001; Mukhtyar et al. 2009), BVAS (version 3) was identically applied to patients with AAV to standardize the scoring method (Yoo et al. 2018).

\section{Assessment of clinical, laboratory data, and serum MBL}

The clinical and laboratory data collected were assessed on the date of serum isolation. Clinical data included AAV variants, age, sex, comorbidities, clinical manifestations based on the items of BVAS, and concurrent medications. Laboratory data included ANCA status, white blood cell and platelet counts, hemoglobin, fasting glucose, blood urea nitrogen, creatinine, total protein, serum albumin, aspartate aminotransferase, alanine aminotransferase, total bilirubin, erythrocyte sedimentation rate (ESR), C-reactive protein (CRP), C3, and C4. Serum MBL level was measured from stored samples only in AAV patients using a commercial enzyme-linked immunosorbent assay (ELISA) kit (Abcam, Cambridge, United Kingdom) according to the manufacturer's instructions.

\section{Statistical analyses}

All statistical analyses were performed using MedCalc statistical software version 19.1 (MedCalc Software bvba, Ostend, Belgium). Continuous variables that were normally distributed in Shapiro-Wilk test were presented as mean \pm standard deviation and as median with interquartile range when non-normally distributed. Differences of serum MBL were compared using Mann-Whitney $U$ and the Kruskal-Wallis test. Categorical variables were presented as frequencies and percentages. Because serum MBL was non-normally distributed and the sample size was small, the correlation coefficient between continuous variables with 
MBL was obtained by Kendall's tau. p values less than 0.05 were considered statistically significant.

Baseline characteristics

A total of $42(52.5 \%), 21(26.3 \%)$, and $17(21.3 \%)$ patients were classified as MPA, GPA, and EGPA, respectively. The median age was 62.0 years and 28 (35.0\%) patients were male. ANCA was detected in 52 patients $(65.0 \%)$, and the most commonly observed clinical manifestation was pulmonary lesions $(63.8 \%)$, followed by renal symptoms $(50.0 \%)$. The median BVAS, VDI, and FFS were $6.5,3.0$, and 1.0 , respectively. In regard to medications, patients were most commonly on treatment with glucocorticoids $(70.0 \%)$, followed by cyclophosphamide and azathioprine (21.3\% and 20.0\%). Among laboratory results, the median ESR and CRP level was $36.5 \mathrm{~mm} / \mathrm{h}$ (normal range $<20$ ) and 2.4 (normal range $<8$ ) $\mathrm{mg} / \mathrm{dL}$. The median value of $\mathrm{C} 3, \mathrm{C} 4$, and serum MBL was 110.8 (normal 90-180) mg/dL, 23.5 (normal 10-40) mg/dL, and $8.6 \mathrm{ng} /$ $\mathrm{mL}$, respectively (Table 1 ).

Correlation between continuous variables and serum $M B L$

In correlation analysis, BVAS was significantly correlated with $\mathrm{ESR}(\mathrm{R}=0.337, \mathrm{p}=0.002)$ and $\mathrm{CRP}(\mathrm{R}=0.366$, $\mathrm{p}<0.001)$. Meanwhile, serum MBL was correlated with BVAS $(\mathrm{R}=0.169, \mathrm{p}=0.027)$, but not with $\mathrm{C} 3$ and $\mathrm{C} 4(\mathrm{p}=$ 0.829 and $p=0.555$ ) (Fig. 1). For clinical subcategories comprising BVAS, serum MBL was found to be significantly associated with the pulmonary manifestation score based on the BVAS $(\mathrm{R}=0.247, \mathrm{p}=0.001)$ (Fig. 2). However, the association between serum MBL with ESR ( $p$ $=0.841)$ and CRP $(p=0.914)$ was not evident. No significant correlation was found between serum MBL and other AAV-specific indices, as well as the remaining laboratory variables. In addition, age was not found to be related to serum MBL (Fig. 1).

Difference of serum MBL according to disease variants, sex, ANCA status, comorbidities, and clinical manifestations

On comparing serum MBL between AAV variants, it was found that patients with GPA had the highest serum MBL (median MBL $9.3 \mathrm{ng} / \mathrm{mL}$ in GPA). However, differences were not found concerning serum MBL according to sex, ANCAs, and comorbidities. In addition, there were no difference in serum MBL according to the presence and absence of clinical manifestations, although serum MBL was found to be slightly higher in patients with pulmonary manifestations compared to those without (median MBL 8.9 $\mathrm{ng} / \mathrm{mL}$ vs. $8.3 \mathrm{ng} / \mathrm{mL}, \mathrm{p}=0.099)$ (Table 2 ).

\section{Discussion}

A recent study reported that high serum MBL level was associated with thrombocytopenia and seizure in patients with systemic lupus erythematosus (SLE), and hypothesized that these results could be attributed to the
Table 1. Baseline characteristics of 80 patients with AAV.

\begin{tabular}{|c|c|}
\hline Variables & Values \\
\hline \multicolumn{2}{|l|}{ Variants of AAV [n, (\%)] } \\
\hline MPA & $42(52.5)$ \\
\hline GPA & $21(26.3)$ \\
\hline EGPA & $17(21.3)$ \\
\hline \multicolumn{2}{|l|}{ Demographic data } \\
\hline Age (year old) & $62.0(21.0)$ \\
\hline Male sex $[\mathrm{n},(\%)]$ & $28(35.0)$ \\
\hline \multicolumn{2}{|l|}{ ANCA status (n, (\%)) } \\
\hline MPO-ANCA (or P-ANCA) positivity & $45(56.3)$ \\
\hline PR3-ANCA (or C-ANCA) positivity & $7(8.8)$ \\
\hline Both ANCA positivity & $0(0)$ \\
\hline ANCA negativity & $28(35.0)$ \\
\hline \multicolumn{2}{|l|}{ Comorbidities (n, $(\%))$} \\
\hline Hypertension & $19(23.8)$ \\
\hline Diabetes mellitus & $15(18.8)$ \\
\hline \multicolumn{2}{|l|}{ Clinical manifestations [n, (\%)] } \\
\hline General & $27(33.8)$ \\
\hline Cutaneous & $9(11.3)$ \\
\hline Mucous membranes/eyes & $5(6.3)$ \\
\hline Ear Nose Throat & $36(45.0)$ \\
\hline Pulmonary & $51(63.8)$ \\
\hline Cardiovascular & $5(6.3)$ \\
\hline Gastrointestinal & $0(0)$ \\
\hline Renal & $40(50.0)$ \\
\hline Nervous & $18(22.5)$ \\
\hline \multicolumn{2}{|l|}{ AAV-specific indices } \\
\hline BVAS & $6.5(10.8)$ \\
\hline VDI & $3.0(2.0)$ \\
\hline FFS & $1.0(1.0)$ \\
\hline SF-36 PCS & $48.6(29.5)$ \\
\hline SF-36 MCS & $57.5(21.1)$ \\
\hline \multicolumn{2}{|l|}{ Concurrent medications (n, $(\%))$} \\
\hline Glucocorticoids & $56(70.0)$ \\
\hline Cyclophosphamide & $17(21.3)$ \\
\hline Azathioprine & $16(20.0)$ \\
\hline Rituximab & $2(2.5)$ \\
\hline Methotrexate & $2(2.5)$ \\
\hline Tacrolimus & $2(2.5)$ \\
\hline Mycophenolate mofetil & $2(2.5)$ \\
\hline \multicolumn{2}{|l|}{ Laboratory results } \\
\hline White blood cell count $\left(/ \mathrm{mm}^{3}\right)$ & $7,685.0(4,460.0)$ \\
\hline Hemoglobin $(\mathrm{g} / \mathrm{dL})$ & $11.8(3.8)$ \\
\hline Platelet $\left(\times 1,000 / \mathrm{mm}^{3}\right)$ & $254.0(135.3)$ \\
\hline Fasting glucose (mg/dL) & $102.0(34.8)$ \\
\hline Blood urea nitrogen $(\mathrm{mg} / \mathrm{dL})$ & $18.9(16.5)$ \\
\hline Creatinine $(\mathrm{mg} / \mathrm{dL})$ & $0.9(1.5)$ \\
\hline Total protein $(\mathrm{g} / \mathrm{dL})^{\pi}$ & $6.4(0.7)$ \\
\hline Serum albumin $(\mathrm{g} / \mathrm{dL})^{\pi}$ & $3.6(0.7)$ \\
\hline Aspartate aminotransferase (IU/L) & $18.0(9.0)$ \\
\hline Alanine aminotransferase (IU/L) & $18.0(12.8)$ \\
\hline Total bilirubin (mg/dL) & $0.5(0.4)$ \\
\hline $\operatorname{ESR}(\mathrm{mm} / \mathrm{h})$ & $36.5(41.5)$ \\
\hline $\mathrm{CRP}(\mathrm{mg} / \mathrm{L})$ & $2.4(14.5)$ \\
\hline $\mathrm{C} 3(\mathrm{mg} / \mathrm{dL})$ & $110.8(29.6)$ \\
\hline $\mathrm{C} 4(\mathrm{mg} / \mathrm{dL})^{\pi}$ & $24.5(9.0)$ \\
\hline Serum MBL $(\mathrm{ng} / \mathrm{mL})$ & $8.6(1.6)$ \\
\hline
\end{tabular}

Values are expressed as median (interquartile range) or in numbers (\%).

$\mathrm{AAV}$, antineutrophil cytoplasmic antibody-associated vasculitis; MPA, microscopic polyangiitis; GPA, granulomatosis with polyangiitis; EGPA, eosinophilic granulomatosis with polyangiitis; ANCA, antineutrophil cytoplasmic antibody; MPO, myeloperoxidase; P, perinuclear; PR3, proteinase 3; C, cytoplasmic; BVAS, birmingham vasculitis activity score; VDI, vasculitis damage index; FFS, five-factor score; SF-36, short form 36-item health survey; PCS, physical component score; MCS, mental component score; ESR, erythrocyte sedimentation rate; $\mathrm{CRP}, \mathrm{C}$-reactive protein; $\mathrm{C} 3$, complement 3; C4, complement 4; MBL, mannose-binding lectin.

"Continuous variables that were normally distributed are expressed as mean \pm standard deviation. 



Fig. 1. Correlation between continuous variables and serum MBL.

Serum MBL was correlated with BVAS, but not with $\mathrm{C} 3$ and $\mathrm{C} 4$. In addition, serum MBL was not associated with ESR, $\mathrm{CRP}$, and age.

The correlation between continuous variables and serum MBL was calculated in 80 patients.

MBL, mannose-binding lectin; BVAS, Birmingham vasculitis activity score; C, complement; ESR, erythrocyte sedimentation rate; $\mathrm{CRP}, \mathrm{C}$-reactive protein.

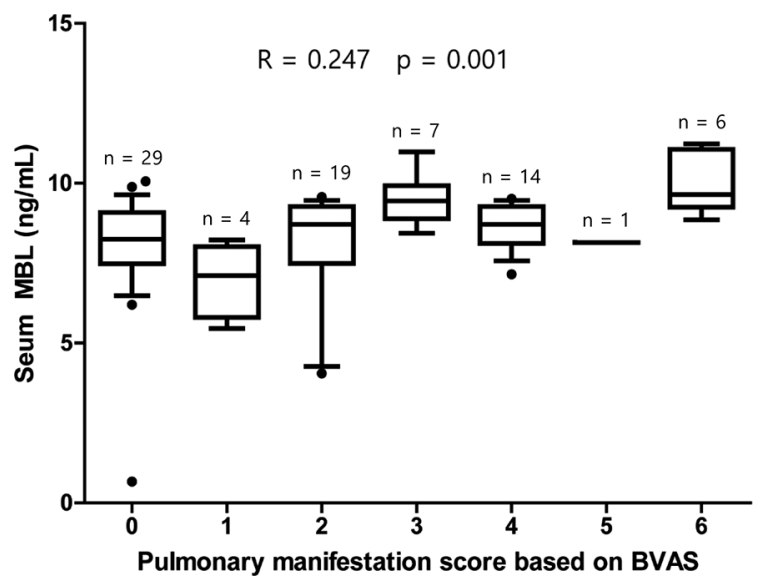

Fig. 2. Correlation between pulmonary manifestation score based on BVAS and serum MBL.

Serum MBL was significantly associated with pulmonary manifestation score based on BVAS.

The error bars indicate $10-90 \%$ values.

MBL, mannose-binding lectin; BVAS, Birmingham vasculitis activity score.

activation of lectin complement pathway (Cieslinski et al. 2018). In this context, the lack of MASP-1 and -3 have been reported to participate in the development of glomerulonephritis in lupus-prone mice (Machida et al. 2018). However, another study showed contradictory results that patients with SLE presented with mild and moderate MBL deficiency more often compared to healthy controls (Perazzio et al. 2016). Likewise, inconsistent results were found concerning the role of serum MBL in other autoimmune and rheumatic diseases. Serum MBL was shown to be associated with pediatric inflammatory bowel diseases but not with the clinical phenotype of patients with Crohn's disease (Kovacs et al. 2013; Kim et al. 2014). In addition, even though serum MBL was not reported to be associated with clinical phenotypes, spondyloarthritis subjects with
MBL deficiency have been shown to exhibit a more serious radiological progression (Aydin et al. 2007; Skare et al. 2017). Taken together, it can be assumed that the role of serum MBL in autoimmune diseases could be variable depending on the disease type, ethnicity, and region.

In the pathogenesis of AAV, circulating ANCAs recognize and bind to the neutrophil-antigens attached to the neutrophil cell membrane. Alternatively, they may form an immune-complex with the released antigens and bind to $\mathrm{Fc} \gamma$ receptors on the neutrophil surface, resulting in the activation of neutrophils (Jennette et al. 2013a; Jennette and Falk 2014). Activated neutrophils have been suggested to promote inflammation in AAV through three different mechanisms. First, neutrophils can migrate into the adjacent tissues by the enhanced interaction with endothelial adhesion molecules. Second, the production and secretion of reactive oxygen radicals by neutrophils could accelerate inflammation. Third, the release of neutrophil granular proteins by degranulated neutrophils could provoke vascular injury (Jennette et al. 2013a). Given that serum MBL is capable of activating the lectin pathway, and the production of $\mathrm{C} 5 \mathrm{a}$ is a crucial factor that sustains the vicious loop of neutrophil activation in AAV (Chen et al. 2017; Hilhorst et al. 2017), it can be hypothesized that serum MBL level is related to disease activity in AAV.

Based on the reasons provided, we speculated that serum MBL might be associated with the disease activity of $\mathrm{AAV}$ as well as the serum concentration of $\mathrm{C} 3$ and $\mathrm{C} 4$. However, correlation analysis showed that serum MBL was not strongly associated with BVAS, whereas serum MBL was not associated with $\mathrm{C} 3$ and $\mathrm{C} 4$. Even though the activation of complement pathway plays an important role in AAV, two hypotheses can be suggested as why serum MBL was not highly correlated with the disease activity of AAV. First, MBL participates in the lectin pathway via the formation of the MBL-MASP complex; however, complement 
Table 2. Comparison of serum MBL according to disease variants, sex, ANCA status, comorbidities, and clinical manifestations.

\begin{tabular}{|c|c|c|}
\hline Variables & Serum MBL level & p value \\
\hline \multicolumn{3}{|l|}{ Variants of AAV } \\
\hline MPA/ GPA/EGPA & $8.4(1.6) / 9.3(0.7) / 8.2(1.4)$ & 0.009 \\
\hline \multicolumn{3}{|l|}{ Sex } \\
\hline Male/Female & $8.8(1.4) / 8.5(1.7)$ & 0.250 \\
\hline \multicolumn{3}{|l|}{ ANCA status } \\
\hline MPO-ANCA (or P-ANCA) $(+) /(-)$ & $8.6(1.4) / 8.6(1.8)$ & 0.782 \\
\hline PR3-ANCA (or C-ANCA) $(+) /(-)$ & $9.4(0.6) / 8.5(1.7)$ & 0.104 \\
\hline \multicolumn{3}{|l|}{ Presence of comorbidities } \\
\hline Hypertension $(+) /$ Hypertension $(-)$ & $8.9(1.4) / 8.6(1.8)$ & 0.400 \\
\hline Diabetes mellitus $(+)^{\pi} /$ Diabetes mellitus $(-)$ & $8.5(0.9) / 8.7(1.7)$ & 0.763 \\
\hline \multicolumn{3}{|l|}{ Presence of clinical manifestations } \\
\hline General (+)/General (-) & $8.9(1.5) / 8.6(1.6)$ & 0.224 \\
\hline Cutaneous $(+) /$ Cutaneous $(-)$ & $8.8(1.3) / 8.6(1.8)$ & 0.813 \\
\hline Mucous membranes/eyes (+)/Mucous membranes/eyes (-) & $8.9(2.0) / 8.6(1.8)$ & 0.361 \\
\hline Ear Nose Throat $(+)^{\top / E} /$ Ear Nose Throat $(-)$ & $8.6(1.3) / 8.6(1.7)$ & 0.471 \\
\hline Pulmonary $(+) /$ Pulmonary $(-)$ & $8.9(1.4) / 8.3(1.6)$ & 0.099 \\
\hline Cardiovascular $(+) /$ Cardiovascular $(-)$ & $8.5(0.7) / 8.6(1.8)$ & 0.889 \\
\hline Renal (+)/Renal (-) & $8.5(1.5) / 8.7(1.6)$ & 0.946 \\
\hline Nervous (+)/Nervous $(-)$ & $8.6(1.8) / 8.7(1.4)$ & 0.600 \\
\hline
\end{tabular}

Values $(\mathrm{ng} / \mathrm{mL})$ are expressed as median (interquartile range).

MBL, mannose-binding lectin; ANCA, antineutrophil cytoplasmic antibody; AAV, antineutrophil cytoplasmic antibody-associated vasculitis; MPA, microscopic polyangiitis; GPA, granulomatosis with polyangiitis; EGPA, eosinophilic granulomatosis with polyangiitis; MPO, myeloperoxidase; $\mathrm{P}$, perinuclear; PR3, proteinase 3; C, cytoplasmic.

"Continuous variables that were normally distributed are expressed as mean \pm standard deviation.

activation could be also mediated through classical and alternative pathway. Second, serum MBL may undergo a relatively rapid turnover, whereas BVAS could reflect a consequence of prolonged inflammation, which presents as nodules or cavities, infiltrate, and subglottic stenosis among pulmonary manifestations. Thus, the disparity of serum MBL and BVAS might be also attributable to the complexity in the scoring method of BVAS.

In addition to disease activity, we further investigated the correlation of serum MBL with BVAS scores of various organ systems. This analysis increases specificity in detecting the severity of individual organ involved. We found that serum MBL was positively correlated with assigned scores for pulmonary manifestations among the nine organbased systems. Even though this finding implies that there might be a link between serum MBL and organ-specific manifestations in $\mathrm{AAV}$, serum MBL level was not different between patients with pulmonary manifestations and without. Additional validation is required in future studies because a direct relationship cannot be demonstrated from the results in this study.

This is the first study to investigate and report the clinical significance of serum MBL in patients with AAV; however, it has certain limitations. First, the number of patients included was not large enough to draw a clear conclusion.
Second, serum MBL level was measured using serum and we failed to measure the levels of circulating $\mathrm{C} 5 \mathrm{a}$, which is a common end-product of the complement pathway. Finally, the difference in the ELISA kits that were used to detect serum MBL in our study might have influenced the study results. Additional investigations with larger numbers of AAV patients are required to overcome these limitations and provide more reliable information regarding the clinical role of serum MBL in AAV.

In conclusion, we demonstrate that serum MBL is correlated with BVAS and pulmonary manifestation score based on the BVAS, even though the correlation with other AAV-specific indices and clinical and laboratory variables was not shown. Future investigations are required to provide more reliable information regarding the clinical role of serum MBL in AAV.

\section{Acknowledgments}

This research was supported by Basic Science Research Program through the National Research Foundation of Korea (NRF) funded by the Ministry of Education (2017R1D1A1B03029050) and a grant from the Korea Health Technology R\&D Project through the Korea Health Industry Development Institute, funded by the Ministry of Health and Welfare, Republic of Korea 
(HI14C1324).

\section{Author Contributions}

Conceptualization, T.Y., S.S.A. and S.W.L.; Data curation, S.S.A., J.Y., B.W.Y., H.C.K. and S.W.L.; Formal analysis, T.Y., S.S.A. and S.W.L.; Methodology, S.M.J., J.J.S. and S.W.L.; Project administration, T.Y. and S.S.A.; Supervision, Y.B.P. and S.W.L.; Writing - original draft, T.Y., S.S.A., and S.W.L; Writing - review and editing, T.Y., S.S.A., J.Y., B.W.Y., H.C.K., S.M.J., J.J.S., Y.B.P. and S. W.L.

\section{Conflict of Interest}

The authors declare no conflict of interest.

\section{References}

Aydin, S.Z., Atagunduz, P., Inanc, N., Bicakcigil, M., Tasan, D., Temel, M. \& Direskeneli, H. (2007) Mannose binding lectin levels in spondyloarthropathies. J. Rheumatol., 34, 20752077.

Bhamra, K. \& Luqmani, R. (2012) Damage assessment in ANCAassociated vasculitis. Curr. Rheumatol. Rep., 14, 494-500.

Chen, M., Jayne, D.R.W. \& Zhao, M.H. (2017) Complement in ANCA-associated vasculitis: mechanisms and implications for management. Nat. Rev. Nephrol., 13, 359-367.

Cieslinski, J.Z., Skare, T.L., Nisihara, R., De Messias-Reason, I.J. \& Utiyama, S.R.R. (2018) Mannose-binding lectin serum levels in patients with systemic lupus erythematosus: association with thrombocytopaenia and seizure. Lupus, 27, 372-379.

Ezekowitz, R.A. (2003) Role of the mannose-binding lectin in innate immunity. J. Infect. Dis., 187 Suppl 2, S335-339.

Guillevin, L., Pagnoux, C., Seror, R., Mahr, A., Mouthon, L. \& Le Toumelin, P.; French Vasculitis Study Group (FVSG) (2011) The Five-Factor Score revisited: assessment of prognoses of systemic necrotizing vasculitides based on the French Vasculitis Study Group (FVSG) cohort. Medicine (Baltimore), 90, 19-27.

Han, C.W., Lee, E.J., Iwaya, T., Kataoka, H. \& Kohzuki, M. (2004) Development of the Korean version of Short-Form 36-Item Health Survey: health related QOL of healthy elderly people and elderly patients in Korea. Tohoku J. Exp. Med., 203, 189-194.

Hilhorst, M., van Paassen, P., van Rie, H., Bijnens, N., HeeringsRewinkel, P., van Breda Vriesman, P., Cohen Tervaert, J.W. \& Limburg Renal, R. (2017) Complement in ANCA-associated glomerulonephritis. Nephrol. Dial. Transplant., 32, 13021313.

Jennette, J.C. \& Falk, R.J. (2014) Pathogenesis of antineutrophil cytoplasmic autoantibody-mediated disease. Nat. Rev. Rheumatol., 10, 463-473.

Jennette, J.C., Falk, R.J., Hu, P. \& Xiao, H. (2013a) Pathogenesis of antineutrophil cytoplasmic autoantibody-associated smallvessel vasculitis. Annu. Rev. Pathol., 8, 139-160.

Jennette, J.C., Falk, R.J., Bacon, P.A., Basu, N., Cid, M.C., Ferrario, F., Flores-Suarez, L.F., Gross, W.L., Guillevin, L., Hagen, E.C., Hoffman, G.S., Jayne, D.R., Kallenberg, C.G., Lamprecht, P., Langford, C.A., et al. (2013b) 2012 revised International Chapel Hill Consensus Conference Nomenclature of Vasculitides. Arthritis Rheum., 65, 1-11.

Kallenberg, C.G. (2014) Key advances in the clinical approach to ANCA-associated vasculitis. Nat. Rev. Rheumatol., 10, 484-493.

Kallenberg, C.G., Stegeman, C.A., Abdulahad, W.H. \& Heeringa, P. (2013) Pathogenesis of ANCA-associated vasculitis: new possibilities for intervention. Am. J. Kidney Dis., 62, 1176-
1187.

Kamesh, L., Heward, J.M., Williams, J.M., Gough, S.C., Savage, C.O. \& Harper, L. (2007) Mannose-binding lectin gene polymorphisms in a cohort study of ANCA-associated small vessel vasculitis. Rheumatology (Oxford), 46, 1076-1078.

Kim, Y.S., Kim, Y.H., Ye, B.D., Park, D.W., Kim, J.W. \& Han, D.S. (2014) Mannose-binding lectin deficiency is not associated with anti-Saccharomyces cerevisiae antibody in Korean Crohn's disease patients. Clin. Chim. Acta, 429, 206-211.

Kovacs, M., Papp, M., Lakatos, P.L., Jacobsen, S., Nemes, E., Polgar, M., Solyom, E., Bodi, P., Horvath, A., Molnar, K., Szabo, D., Cseh, A., Muller, K.E., Dezsofi, A., Arato, A., et al. (2013) Low mannose-binding lectin (MBL) is associated with paediatric inflammatory bowel diseases and ileal involvement in patients with Crohn disease. J. Crohns Colitis, 7, 134-141.

Leavitt, R.Y., Fauci, A.S., Bloch, D.A., Michel, B.A., Hunder, G.G., Arend, W.P., Calabrese, L.H., Fries, J.F., Lie, J.T., Lightfoot, R.W. Jr., Mcshane, D.j., Mills, J.A., Stevens, M.B., Wallace, S.L. \& Zvaifler, N.J. (1990) The American College of Rheumatology 1990 criteria for the classification of Wegener's granulomatosis. Arthritis Rheum., 33, 1101-1107.

Machida, T., Sakamoto, N., Ishida, Y., Takahashi, M., Fujita, T. \& Sekine, H. (2018) Essential roles for mannose-binding lectinassociated serine protease- $1 / 3$ in the development of lupuslike glomerulonephritis in MRL/lpr mice. Front. Immunol., 9, 1191

Masi, A.T., Hunder, G.G., Lie, J.T., Michel, B.A., Bloch, D.A., Arend, W.P., Calabrese, L.H., Edworthy, S.M., Fauci, A.S., Leavitt, R.Y., Lightfoot, R.W. Jr., Mcshane, D.J., Mills, J.A., Stevesn, M.B., Wallace, S.L. \& Zvaifler, N.J. (1990) The American College of Rheumatology 1990 criteria for the classification of Churg-Strauss syndrome (allergic granulomatosis and angiitis). Arthritis Rheum., 33, 1094-1100.

Mukhtyar, C., Lee, R., Brown, D., Carruthers, D., Dasgupta, B., Dubey, S., Flossmann, O., Hall, C., Hollywood, J., Jayne, D., Jones, R., Lanyon, P., Muir, A., Scott, D., Young, L., et al. (2009) Modification and validation of the Birmingham Vasculitis Activity Score (version 3). Ann. Rheum. Dis., 68, 18271832.

Pagowska-Klimek, I. \& Cedzynski, M. (2014) Mannan-binding lectin in cardiovascular disease. Biomed Res. Int., 2014, 616817.

Perazzio, S.F., Silva, N.P., Carneiro-Sampaio, M. \& Andrade, L.E. (2016) Mild and moderate Mannose Binding Lectin deficiency are associated with systemic lupus erythematosus and lupus nephritis in Brazilian patients. Rev. Bras. Reumatol. Engl. Ed., 56, 220-227.

Roos, A., Daha, M.R., van Pelt, J. \& Berger, S.P. (2007) Mannosebinding lectin and the kidney. Nephrol. Dial. Transplant., 22, 3370-3377.

Skare, T.L., Nisihara, R., Cieslinski, J.Z., Zeni, J.O., Rasera, H.N., Messias-Reason, I. \& Utiyama, S.R. (2017) Mannose-binding lectin deficiency in Brazilian patients with spondyloarthritis. Immunol. Invest., 46, 183-189.

Stone, J.H., Hoffman, G.S., Merkel, P.A., Min, Y.I., Uhlfelder, M.L., Hellmann, D.B., Specks, U., Allen, N.B., Davis, J.C., Spiera, R.F., Calabrese, L.H., Wigley, F.M., Maiden, N., Valente, R.M., Niles, J.L., et al. (2001) A disease-specific activity index for Wegener's granulomatosis: modification of the Birmingham Vasculitis Activity Score. International Network for the Study of the Systemic Vasculitides (INSSYS). Arthritis Rheum., 44, 912-920.

Takahashi, K. \& Ezekowitz, R.A. (2005) The role of the mannosebinding lectin in innate immunity. Clin. Infect. Dis., 41 Suppl 7, S440-444.

Turner, M.W. (2003) The role of mannose-binding lectin in health and disease. Mol. Immunol., 40, 423-429.

Watts, R., Lane, S., Hanslik, T., Hauser, T., Hellmich, B., Koldingsnes, W., Mahr, A., Segelmark, M., Cohen-Tervaert, J.W. \& 
Scott, D. (2007) Development and validation of a consensus methodology for the classification of the ANCA-associated vasculitides and polyarteritis nodosa for epidemiological studies. Ann. Rheum. Dis., 66, 222-227.

Yoo, J., Ahn, S.S., Jung, S.M., Song, J.J., Park, Y.B. \& Lee, S.W. (2018) Delta Neutrophil Index is associated with vasculitis activity and risk of relapse in ANCA-associated vasculitis. Yonsei Med. J., 59, 397-405.

Yuan, J., Gou, S.J., Huang, J., Hao, J., Chen, M. \& Zhao, M.H. (2012) C5a and its receptors in human anti-neutrophil cytoplasmic antibody (ANCA)-associated vasculitis. Arthritis Res. Ther., 14, R140. 